\title{
On the central limit theorem and iterated logarithm law for stationary processes.
}

\author{
C.C. Heyde
}

It has recently emerged that a convenient way to establish central limit and iterated logarithm results for processes with stationary increments is to use approximating martingales with stationary increments. Functional forms of the limit results can be obtained via a representation for the increments of the stationary process in terms of stationary martingale differences plus other terms whose sum telescopes and disappears under suitable norming. Results based on the most general form of such a representation are here obtained.

\section{Introduction and principal results}

In this paper our setting is that of a probability space $(\Omega, B, P)$ with an ergodic one-to-one bimeasurable measure preserving transformation $T$. Let $L_{2}(P)$ be the Hilbert space of random variables with finite second moment. Define $U$ on $L_{2}(P)$ by $U X(\omega)=X(T \omega)$ for $X \in L_{2}(P)$, $\omega \in \Omega$, and write $X_{k}=U^{k} X_{0}$ for some particular $X_{0} \in L_{2}(P)$ with $E X_{0}=0$. Set also, $S_{0}=0, S_{n}=\sum_{k=1}^{n} x_{k}$ for $n \geq 1$ and $\sigma_{n}^{2}=E S_{n}^{2}$. We shall be concerned with giving invariance principles for both the central limit and iterated logarithm law for appropriate random functions

Received 3 September 1974. 
in $C$ or $D$ defined from the partial sums $s_{k}$. Here $C=C[0,1]$ is the space of continuous functions on $[0,1]$ and $D=D[0,1]$ is the space of continuous functions on $[0,1]$ which are right continuous with left hand limits. In both cases we employ the supremum metric which we denote by $\rho$.

Let $\left\{\theta_{n}(\cdot)\right\}$ be a sequence of random functions on $[0,1]$ defined by $\theta_{n}(t)=s_{j} / \sigma_{n}, j / n \leq t<(j+1) / n, j=0,1, \ldots, n-1$,

and

$$
\theta_{n}(1)=S_{n} / \sigma_{n}
$$

Also, let $\left\{\eta_{n}(\cdot)\right\}$ be a sequence of random functions on $[0,1]$ defined by

$n_{n}(t)=\left(2 \sigma_{n}^{2} \log \log \sigma_{n}^{2}\right)^{-\frac{1}{2}}\left(s_{i}+(n t-k) X_{k}\right)$,

$$
k \leq n t \leq k+1, k=0,1, \ldots, n-1 .
$$

Let $K$ be the set of absolutely continuous $x \in C$ such that

$$
x(0)=0
$$

and

$$
\int_{0}^{1}[\dot{x}(t)]^{2} d t \leq 1
$$

where $\dot{x}$ denotes the derivative of $x$ determined almost everywhere with respect to Lebesgue measure. Also define

$$
g=\sup \left\{n: \sigma_{n}^{2} \leq e\right\} \text {. }
$$

Let $F_{0}$ be a $\sigma$-field such that $F_{0} \subset B$ and $F_{0} \subset T^{-1}\left(F_{0}\right)$ and write $F_{k}=T^{-k}\left(F_{0}\right), F_{-\infty}=\bigcap_{k=-\infty}^{\infty} F_{k}$ and $F_{\infty}=\sigma$-field generated by $\underset{k=-\infty}{\bigcup} F_{k}$. Our object is the following theorem.

THEOREM. If 


$$
\sum_{n=1}^{\infty}\left\{\overline{1 i m}_{m \rightarrow \infty} E\left(\sum_{r=n}^{m} x_{r}\right)^{2}+\overline{\lim }_{m \rightarrow \infty} E\left(\sum_{r=n}^{m} x_{-r}\right)^{2}\right\}<\infty,
$$

where

$$
X_{r}=E\left(X_{-r} \mid F_{0}\right)-E\left(X_{-r} \mid F_{-1}\right),
$$

and

(2) $E\left(X_{0} \mid F_{\infty}\right)=X_{0}$ almost surely, $E\left(X_{0} \mid F_{-\infty}\right)=0$ almost surely,

then $\lim _{n \rightarrow \infty} \sigma_{n} / \sqrt{n}=\sigma$ exists for $0 \leq \sigma<\infty$. If $\sigma>0$ then $\theta_{n} \stackrel{d}{\longrightarrow} w$ in the sense $(D, \rho)$ where $W$ is a standard Wiener process. Also, $g<\infty$, $\left\{n_{n} ; n>g\right\}$ is relatively compact and the set of $i t s$ limit points coincides with $K$.

This result extends those of Theorem 3 of Scott [5] (central limit case) and Theorem 2 of Heyde and Scott [3] (iterated logarithm case) which were both given with the condition

$$
\sum_{m=1}^{\infty}\left\{\left(E\left[E\left(X_{0} \mid F_{-m}\right)\right]^{2}\right)^{\frac{1}{2}}+\left(E\left[X_{0}-E\left(X_{0} \mid F_{m}\right)\right]^{2}\right)^{\frac{1}{2}}\right\}<\infty
$$

replacing those above. The idea in each case involves a representation for the $X^{\prime} s$ of the form

$$
X_{0}=Y_{0}+U Z_{0}-z_{0}
$$

where the $Y_{0}, Z_{0} \in L_{2}(P)$ and $\left\{U^{k_{Y}}\right\}$ forms a stationary ergodic sequence of martingale differences. The martingale approximation idea is due to Gordin [1] and the conditions of the theorem appear to be the most general under which (4) will hold as above. Of course the limit behaviour of $S_{n}$ is then easy to study via the corresponding behaviour of $\sum_{k=1}^{n} U^{k} Y_{0}$, since

$$
\dot{s}_{n}=\sum_{k=1}^{n} U^{k} X_{0}=\sum_{k=1}^{n} U^{k} Y_{0}+U^{n+1} z_{0}-U z_{0}
$$

and the effect of $U^{n+1} z_{0}-U Z_{0}$ disappears under suitable norming. It 
should be remarked that $\overline{\lim }_{n \rightarrow \infty} E\left(\sum_{k=1}^{n} U^{k}\left(X_{0}-Y_{0}\right)\right)^{2}<\infty$ for $Y_{0} \in L_{2}(P)$ with $E\left(Y_{0} \mid F-1\right)=0$ almost surely, if and only if $X_{0}-Y_{0}$ is representable in the form (4) (via Theorem 18.2.2 of Ibragimov and Linnik [4]).

That the representation (4) holds under the asserted conditions of the theorem can easily be extracted from the proof of Theorem 3 of [5].

Condition (1) gives

$$
\lim _{n \rightarrow \infty} \overline{\lim }_{m \rightarrow \infty} E\left(\sum_{r=n}^{m} x_{r}\right)^{2}=\lim _{n \rightarrow \infty} \prod_{m \rightarrow \infty} E\left(\sum_{r=n}^{m} x_{-r}\right)^{2}=0
$$

so that $\sum_{r=0}^{\infty} x_{r}$ and $\sum_{r=0}^{\infty} x_{-r}$ converge in $L_{2}(P)$. Then

$$
\varlimsup_{m \rightarrow \infty} E\left(\sum_{r=n}^{m} x_{r}\right)^{2}+\overline{\lim }_{m \rightarrow \infty} E\left(\sum_{r=n}^{m} x_{-r}\right)^{2}=E\left(\sum_{r=n}^{\infty} x_{r}\right)^{2}+E\left(\sum_{r=n}^{\infty} x_{-r}\right)^{2},
$$

and our condition (1) is just the condition (46) of [5]. Once the representation (4) is obtained the remainder of the proof is exactly that of Theorem 3 of [5] in the central limit case and Theorem 2 of [3] in the case of the iterated logarithm law.

We remark that $(I)$ arises via the restriction $z_{0} \in L_{2}(P)$. In fact, from [5], p. 133,

$$
E Z_{0}^{2}=\sum_{n=1}^{\infty} E\left\{\left(\sum_{r=n}^{\infty} x_{r}\right)^{2}+\left(\sum_{r=n}^{\infty} x_{-r}\right)^{2}\right\}
$$

Note also that under the conditions of the theorem we have that (4) holds and $\lim _{n \rightarrow \infty} \sigma_{n} / \sqrt{n}=\sigma$ exists for $0 \leq \sigma<\infty$. Now $z_{0} \in L_{2}(P)$, so $\sigma^{2}=E Y_{0}^{2}$, and we can only obtain $\sigma=0$ in the case where $x_{n}=v^{n+1} z_{0}-U^{n} z_{0}$, some $z_{0} \in L_{2}(P)$.

We shall show that a useful sufficient condition for (1), in the presence of (2) is

$$
\sum_{n=1}^{\infty} \sum_{k=0}^{\infty}\left|E\left(X_{k} E\left(X_{0} \mid F-n\right)\right)+E\left(X_{-k} X_{0}\right)-E\left(X_{-k} E\left(X_{0} \mid F_{n-1}\right)\right)\right|<\infty .
$$


To obtain this we first note that

(6) $E\left\{\left(\sum_{r=n}^{m} x_{r}\right)^{2}+\left(\sum_{r=n}^{m} x_{-r}\right)^{2}\right\}=$

$$
=\sum_{r=n}^{m} E\left(x_{r}^{2}+x_{-r}^{2}\right)+2 \sum_{k=1}^{m-n} \sum_{r=n}^{m-k} E\left(x_{r} x_{r+k}+x_{-r^{2}-r-k}\right) .
$$

Now for any $k, r$,

$$
\begin{aligned}
E\left(x_{r} x_{r+k}\right) & =E\left(E\left(X_{-r} \mid F_{0}\right) E\left(X_{-r-k} \mid F_{0}\right)\right)-E\left(E\left(X_{-r} \mid F_{-1}\right) E\left(X_{-r-k} \mid F_{0}\right)\right) \\
& =E\left(X_{-r-k} E\left(X_{-r} \mid F_{0}\right)\right)-E\left(X_{-r-k} E\left(X_{-r} \mid F_{-1}\right)\right) \\
& =E\left(X_{-k} E\left(X_{0} \mid F_{r}\right)\right)-E\left(X_{-k} E\left(X_{0} \mid F_{r-1}\right)\right),
\end{aligned}
$$

so that for $k \geq 0$,

$$
\begin{aligned}
& \sum_{r=n}^{m-k} E\left(x_{r} x_{r+k}\right)=E\left(X_{-k} E\left(X_{0} \mid F_{m-k}\right)\right)-E\left(X_{-k} E\left(X_{0} \mid F_{n-1}\right)\right), \\
& \sum_{r=n}^{m-k} E\left(x_{-r^{2}-r-k}\right)=E\left(X_{k} E\left(X_{0} \mid F_{-n}\right)\right)-E\left(X_{k} E\left(X_{0} \mid F_{-m+k-1}\right)\right) .
\end{aligned}
$$

Standard martingale results give $E\left(X_{0} \mid F_{-n}\right) \stackrel{L_{2}}{\longrightarrow} E\left(X_{0} \mid F_{-\infty}\right)=0$ almost surely, and $E\left(X_{0} \mid F_{n}\right) \stackrel{L_{2}}{\longrightarrow} E\left(X_{0} \mid F_{\infty}\right)=X_{0}$ almost surely, so that $E\left(E\left(X_{0} \mid F_{-n}\right)\right)^{2} \rightarrow 0$ and $E\left(X_{0}-E\left(X_{0} \mid F_{n}\right)\right)^{2} \rightarrow 0$ as $n \rightarrow \infty$, and hence

$$
\lim _{m \rightarrow \infty} \sum_{r=n}^{m} E\left(x_{r}^{2}+x_{-r}^{2}\right)=E X_{0}^{2}-E\left(X_{0} E\left(X_{0} \mid F_{n-1}\right)\right)+E\left(X_{0} E\left(X_{0} \mid F_{-n}\right)\right)
$$

Also,

$$
\begin{aligned}
& \sum_{k=1}^{m-n} \sum_{r=n}^{m-k} E\left(x_{r^{2}} x_{p+k}+x-r^{x}-r-k\right) \\
& =\sum_{k=1}^{m-n}\left\{E\left(x_{k} E\left(x_{0} \mid F_{-n}\right)\right)+E\left(x_{-k} X_{0}\right)-E\left(x_{-k} E\left(x_{0} \mid F_{n-1}\right)\right)\right\} \\
& -\sum_{k=1}^{m-n}\left\{E\left(x_{k} E\left(x_{0} \mid F_{-m+k-1}\right)\right)+E\left(X_{-k} X_{0}\right)-E\left(X_{-k} E\left(x_{0} \mid F_{m-k}\right)\right)\right\}
\end{aligned}
$$

and under the condition (5), 
(8) $\lim _{m \rightarrow \infty} \sum_{k=1}^{m-n} \sum_{r=n}^{m-k} E\left(x_{r} x_{r+k}+x_{-r} x_{-r-k}\right)$

$$
=\sum_{k=1}^{\infty}\left\{E\left(X_{k} E\left(X_{0} \mid F_{-n}\right)\right)+E\left(X_{-k} X_{0}\right)-E\left(X_{-k} E\left(X_{0} \mid F_{n-1}\right)\right)\right\} .
$$

It is then clear from (6), (7), and (8), that (1) holds under the condition (5).

Conditions (1) and (5) do not appear to simplify in any really convenient way in general. However, we remark that we are free to choose a convenient $F_{0}$. For example, if $F_{0}$ is the $\sigma$-field generated by $X_{k}$, $k \leq 0$, then the condition (5) becomes just

$$
\sum_{n=1}^{\infty} \sum_{k=0}^{\infty}\left|E\left(X_{k} E\left(X_{0} \mid F_{-n}\right)\right)\right|<\infty .
$$

In some cases it may be useful to translate (1) (or (5)) into a condition on the Fourier coefficients of the expansion of $X_{0}$ in terms of a suitable complete orthonormal set. Certainly (I) represents a significant improvement over (3) and this justifies its use. An example is given in Section 2 to illustrate this point. It should also be remarked that conditions (1) (or (5)) and (3) provide a convenient vehicle for the study of central limit and iterated logarithm results for stationary processes satisfying mixing conditions. Illustrations concerning uniform mixing processes have been given in the papers [3] and [5].

\section{An example}

To illustrate the improvement of (1) over (2) we introduce the stationary linear process $\{x(n)\}$ given by

$$
x(n)-\mu=\sum_{j=-\infty}^{\infty} \alpha(j) \varepsilon(n-j), \sum_{j=-\infty}^{\infty} \alpha^{2}(j)<\infty,
$$

where the $\varepsilon(n)$ are independent and identically distributed with zero mean and variance $\sigma^{2}$. One widely applicable model which gives rise to this process is the standard version of the mixed autoregression and moving average process. Another is the stationary gaussian process with absolutely continuous spectral density. 
Suppose that $x(1), x(2), \ldots, x(N)$ is a sample of $N$ consecutive observations on the process $\{x(n)\}$ and $\bar{x}$ denotes the sample mean. It follows from the ergodic theorem that $\bar{x} \rightarrow \mu$ almost surely, as $N \rightarrow \infty$, and it is of interest to obtain functional central limit and iterated logarithm results which give information on the rate of this convergence. Here we have $x_{k}=x(k)-\mu$ and we can take $F_{k}$ as the $\sigma$-field generated by $\varepsilon(m), m \leq k$. Then

$$
x_{r}=E\left(X_{-r} \mid F_{0}\right)-E\left(X_{-r} \mid F_{-1}\right)=\alpha(-r) \varepsilon(0),
$$

and the theorem of this paper applies if

$$
\sum_{n=1}^{\infty}\left\{\left(\sum_{r=n}^{\infty} \alpha(r)\right)^{2}+\left(\sum_{r=n}^{\infty} \alpha(-r)\right)^{2}\right\}<\infty .
$$

On the other hand, the corresponding results based on the use of condition (3) hold if

$$
\sum_{n=1}^{\infty}\left[\sum_{r \mid \geq n} \alpha^{2}(r)\right]^{\frac{1}{2}}<\infty,
$$

as in the lemma of Heyde [2]. The condition (10) represents a significant improvement over (11) in the case where the $\alpha$ 's vary continually in sign. For example, $\alpha(|r|)=(-1)^{r} r^{-1}, r \geq 1$. If the $\alpha^{\prime}$ 's are ultimately all positive, an example where (10) holds but (11) does not is provided by $\alpha(|r|) \sim C r^{-3 / 2}(\log r)^{-1}$ as $r \rightarrow \infty$ for some $C>0$.

\section{References}

[1] M.l. Gordin, "The central limit theorem for stationary processes", Soviet Math. Dokz. 10 (1969), 1174-1176.

[2] C.C. Heyde, "An iterated logarithm result for autocorrelations of a stationary linear process", Ann. Probability 2 (1974), 328-332.

[3] C.C. Heyde and D.J. Scott, "Invariance principles for the law of the iterated logarithm for martingales and processes with stationary increments", Ann. Probability 1 (1973), 428-436. 
[4] I.A. Ibragimov and Yu.V. Linnik, Independent and stationary sequences of random variables (Walters-Noordhoff, Groningen, 1971).

[5] D.J. Scott, "Central limit theorems for martingales and for processes with stationary increments using a Skorokhod representation approach", Adv. Appl. Probability 5 (1973), 119-137.

Department of Statistics,

Faculty of Economics,

Australian National University,

Canberra, ACT. 